\title{
Deutsches auch und italienisches anche im Vergleich.
}

\section{Gemeinsame Funktion und sprachspezifischer Gebrauch*}

\author{
Manuela Caterina Moroni (Bergamo) und Ermenegildo Bidese (Trento)
}

\begin{abstract}
The focus of our contribution is the German particle auch 'also' and its Italian counterpart anche. From a semantic point of view, auch and anche convey the same additive meaning in both languages: through them an element, called 'domain of association', is added to a list of further elements for which the auch/anche phrase applies. From a syntactical point of view, auch and anche can occupy different positions in the sentence by modifying the type and the scope of the domain of association. This results in four basic usage patterns which are similar in both languages and correspond to just as many functions of auch/anche: (i) additivity, (ii) sentence connection, (iii) focus domain, and (iv) modality. Despite these common functions, auch and anche interact with the syntax and the information structure of the sentence in German and in Italian differently. Furthermore, they are used in conversation contexts, which are distinctive in both languages.

By starting from the current description of auch and anche in the literature, the first aim of our contribution is to provide evidence for the above-mentioned four basic functions despite their language-specific usage contexts. As a second aim, we want to validate our systematization through the analysis of spoken data for German and Italian, explaining why some functions are more likely to be found in the former than in the latter.
\end{abstract}

\section{$1 \quad$ Einleitung}

Im Mittelpunkt unseres Beitrags stehen die deutsche und italienische additive Partikel auch bzw. anche. Diese gelten für beide Sprachen als „basic additive particles“ (Benazzo/Dimroth 2015: 9). Lexikalisch geht man in der Regel von einer direkten Entsprechung beider Partikeln aus. So geben zweisprachige Wörterbücher als ersten Übersetzungseintrag für auch bzw. anche durchgängig die jeweils andere Partikel an ${ }^{1}$. In beiden Sprachen existieren weitere Elemente, die Additivität ausdrücken können, wie zum Beispiel ebenso, ebenfalls, außerdem, zudem im

\footnotetext{
* Der Aufsatz ist das Ergebnis einer engen Zusammenarbeit zwischen den beiden Autoren. Die wissenschaftliche Verantwortung wird folgendermaßen zugewiesen: Manuela Caterina Moroni für die Kapitel 2.4, 3.1, 3.2, 3.3; Ermenegildo Bidese für die Kapitel 1, 2.1-2.3, 2.5 und 4. Die Autoren danken Giorgia Pigarella für ihre wertvolle Hilfe bei der Aufbereitung der Daten der Korpusanalyse zu auch (Kapitel 3.1). Wir möchten uns bei zwei anonymen Gutachtern für wertvolle Verbesserungsvorschläge bedanken.

${ }^{1}$ Siehe zum Beispiel den Eintrag in Pons Dizionario Online s. v. anche, sowie in Langenscheidt Dizionari online, in Corriere della sera dizionario online und in Dizionario Hoepli Tedesco-Italiano.
} 
Deutschen und pure, inoltre und ancora im Italienischen. Aufgrund der vorrangigen Entsprechung von auch mit anche und umgekehrt und deren ausgeprägter Multifunktionalität, die die anderen additiven Ausdrücke nicht haben, konzentrieren wir uns jedoch in dieser Arbeit auf diese beiden Formen.

Aus semantischer Sicht teilen beide Partikeln grundsätzlich dieselbe additive Grundbedeutung: auch/anche zeigen an, dass es zu einem Element mindestens eine weitere Alternative gibt bzw. dass ein Element zu einer Liste weiterer Elemente gehört, für die der auch/anche-Satz zutrifft (cf. Andorno 2000: $67 \mathrm{zu}$ anche und Dimroth 2004: $25 \mathrm{zu}$ auch). Dazu vergleiche man folgende Beispiele aus Benazzo/Dimroth (2015: 10):

(Context: John recently bought a new house)

(Benazzo/Dimroth 2015: 10)

(1) a. Johannes ist auch [in Urlaub gefahren]. ${ }^{2}$

b. Gianni è anche [andato in vacanza].

In diesem Beispielpaar zeigen auch/anche an, dass zu der von Johannes/Giovanni bereits durchgeführten Tätigkeit (ein neues Haus kaufen), eine weitere hinzukommt, nämlich in Urlaub fahren bzw. andare in vacanza. In Urlaub fahren sprich andare in vacanza gehören also zu der Liste der Tätigkeiten, die Johannes/Giovanni durchführen. Vom Kontext abhängig können auch/anche eine skalare Lesart aufweisen. In diesem Fall entsprechen sie jeweils sogar und addirittura. Der Unterschied zwischen der reinen additiven und der skalaren Lesart besteht darin, dass im zweiten Fall das hinzugefügte Element als unerwartet empfunden wird.

Das durch auch/anche addierte Element wird „Bezugsbereich“ (Dimroth 2004), bzw. „domain of association“ (De Cesare 2015) oder ,added constituent“ (Reis/Rosengren 1997) genannt und kann unterschiedlicher Komplexität sein, wie an folgenden Beispielen zu sehen ist:

(2) a. Marys Absage auch [des frühen Termins] $]_{\mathrm{BB}}$ ist nicht möglich. (leicht abgeändert aus Kleemann 2007: 100)

b. 'La disdetta di Mary anche [dell'appuntamento di mattina] $]_{\mathrm{BB}}$ non è possibile.' (Übersetzung von (2a) von uns: $\mathrm{MM}$ und $\mathrm{EB})^{3}$

(3) a. Peter hat auch [dem Paul ein Buch gekauft $]_{\mathrm{BB}}$ (und nicht nur dir das Essen bezahlt). (Reis/Rosengren 1997: 241).

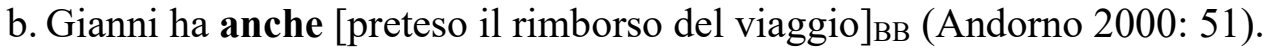

In den Beispielen (2a)-(2b) besteht der Bezugsbereich (im Folgenden in BB abgekürzt) aus einer internen Konstituente der Nominalphrase mit Subjektfunktion. In (3a)-(3b) umfasst der BB die gesamte Verbalphrase.

Obwohl auch und anche dieselbe additive Grundbedeutung teilen, interagieren sie in beiden Sprachen auf unterschiedliche Weise mit der Syntax und der Informationsstruktur des Satzes und tauchen auch in unterschiedlichen pragmatischen Kontexten auf. Die meisten Studien konzentrieren sich gerade auf diese Differenzen im Gebrauch der Partikeln (aus einer kontrastiven Perspektive siehe De Cesare 2015 und Benazzo/Dimroth 2015), indem sie eine

\footnotetext{
${ }^{2}$ In diesen wie in den folgenden Beispielsätzen stammen die Hervorhebungen von uns (MM und EB).

${ }^{3}$ Im Allgemeinen treten auch und anche innerhalb einer DP selten auf. Authentische Belege sind hierfür in Korpora schwer zu finden. Zur Veranschaulichung haben wir den deutschen Beleg ins Italienische übersetzt.
} 
Herangehensweise bevorzugen, die von der Form ausgehen. Aus unserer Sicht ist für den Sprachvergleich demgegenüber eine Herangehensweise geeigneter, die von der Funktion herrührt. Somit kann zunächst von den strukturellen, sprachspezifischen Unterschieden abgesehen werden, um die eigene Leistung der Partikel zu ermitteln. In einem zweiten Schritt lassen sich die Realisierungsmöglichkeiten der ermittelten Funktionen in der jeweiligen Sprache genauer umkreisen. Daher besteht das erste Ziel unserer Analyse darin, die Ergebnisse der Forschungsliteratur zu auch und anche aus einer funktionsgeleiteten Perspektive heraus zu systematisieren. Dabei wird sich zeigen, dass auch und anche trotz der jeweiligen sprachspezifischen Gebrauchskontexte dieselben vier Hauptfunktionen realisieren ${ }^{4}$. Darüber hinaus lassen sich diese auf einem Kontinuum zunehmender grammatischer Abstraktion verteilen. Als zweites Ziel setzen wir uns die Validierung unserer Systematisierung anhand von Daten der gesprochenen Sprache.

Der Beitrag ist wie folgt strukturiert. Im nächsten Teil werden die oben genannten vier Hauptfunktionen von auch und anche anhand der Daten aus der Literatur präsentiert (siehe Kapitel 2). Dabei besprechen wir Gemeinsamkeiten und Unterschiede in den zwei Sprachen anhand der von uns vorgeschlagenen funktionsgeleiteten Perspektive. Im dritten Teil überprüfen wir, inwieweit sich unsere Systematisierung durch Daten der gesprochenen Sprache bestätigen lässt (siehe Kapitel 3). Der vierte Teil fasst die Ergebnisse zusammen und bietet einen Ausblick für zukünftige Untersuchungen (siehe Kapitel 4).

\section{Funktionen von auch und anche}

Die in der Einleitung erläuterte additive Grundbedeutung von auch/anche wird verwendet, um folgende vier Funktionen zu realisieren, die in beiden Sprachen gleich sind:

(i) Additivität

(ii) Satzverknüpfung

(iii) Fokusdomäne

(iv) Modalität

Im Folgenden gehen wir auf jede Funktion ein.

\subsection{Additivität}

Die Additivität ist die prototypische Funktion von auch/anche. Beispiele für diese Funktion sind (4a)-(4b):

(4) a. (Hans und Anna haben bei Lucie angerufen.) (Pasch et al. 2003: 577)

Auch [Peter]BB hat bei Lucie angerufen.

b. (Mr. Blue goes to bed) (Benazzo/Paykin 2017: 271)

Anche [il sig. Rossi]BB va a dormire.

\footnotetext{
${ }^{4}$ In einem Gutachten wird darauf hingewiesen, dass ein umgekehrtes Vorgehen, i. e. vom Korpus zur Funktion zum Sprachvergleich, sinnvoller wäre. Da jedoch sowohl in der kontrastiven als auch in der sprachspezifischen Forschungsliteratur die von uns besprochenen vier Funktionen bereits herausgearbeitet wurden, ist aus unserer Sicht weiterhin plausibel, diese vier Funktionen zu übernehmen, sie zu systematisieren und anhand von Korpusdaten aus beiden Sprachen zu verifizieren. Das ermöglicht einen stichhaltigen Vergleich zwischen deutschem auch und italienischem anche.
} 
In dieser Funktion bezieht sich die Partikel auf ihren BB. In (4a)-(4b) ist dieser die Subjektkonstituente. Stehen auch und anche im Mittelfeld bzw. zwischen den Elementen des Verbalkomplexes, wie in (5a)-(5b), muss die Reichweite des BBs durch den Kontext desambiguiert werden:

(5) a. Peter hat auch [[bei Lucie $]_{\mathrm{BB} 1}$ angerufen $]_{\mathrm{BB} 2}$

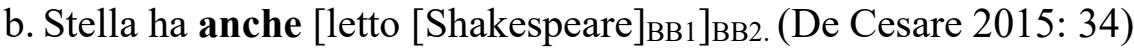

Je nach Kontext kann in (5a) der BB nur bei Lucie oder die ganze Verbalphrase umfassen. Analog dazu kann in (5b) der BB entweder nur Shakespeare oder letto Shakespeare sein. In der additiven Funktion sind auch und anche in der Regel ihrem BB unmittelbar vorangestellt. In Nachstellung gilt auch demgegenüber als ungrammatisch (cf. Pasch et al. 2003: 577):

(6) *[Meine Freundin in Australien $]_{\mathrm{BB}}$ auch hat davon gehört.

Was das Italienische betrifft, könnte aus unserer Sicht die Nachstellung akzeptiert werden, vor allem mit leichten Bezugskonstituenten wie in (7):

(7) Sprecher A: ha comprato quaderni e penne

Sprecher B: ?[il libro] $]_{\mathrm{BB}}$ anche ha comprato

,A: (Er) hat Hefte und Stifte gekauft B: Das Buch auch hat (er) gekauft.

Im Hinblick auf die Informationsstruktur bildet der BB der Partikel den Fokus des Satzes. Wird die Partikel weggelassen, bleibt die Fokus-Hintergrund-Gliederung des Satzes unverändert, wobei die Additivität verloren geht. Da sich auch und anche auf den Fokus beziehen, werden sie der Literatur teilweise als Fokuspartikeln (Dimroth 2004) bzw. „(avverbi) focalizzatori“ (Andorno 1999, 2000; Ricca 1999; Borreguero Zoulaga 2012) bezeichnet.

\subsection{Satzverknüpfung}

Der BB von auch und auche kann auch einen ganzen Satz umfassen. In diesem Fall verknüpfen die Partikeln ihren Bezugsbereich bzw. den Satz, in dem sie sich befinden, mit dem vorangegangenen und werden somit der Funktionsklasse der Konnektoren bzw. congiunzioni zugeordnet. Man vergleiche folgendes Beispiel aus Pasch et al. (2003: 24):

(8) Dann verfasste er über seine Erkenntnisse den Nischnij Nowgoroder Prolog und machte für die Gegend kostenlose Reklame. Auch [erreichte er eine Vereinfachung des Registrierungsverfahrens] $]_{\text {BB. }}$ (Siegl 1997: 93)

In (8) tritt auch alleine im Vorfeld auf und hat adverbiale Funktion. Als satzverknüpfendes Element kann die Partikel genauso im Mittelfeld erscheinen, was in (9) deutlich wird:

(9) Dann verfasste er über seine Erkenntnisse den Nischnij Nowgoroder Prolog und machte für die Gegend kostenlose Reklame. [Er erreichte auch eine Vereinfachung des Registrierungsverfahrens $]_{\mathrm{BB}}$.

Was die Partikel anche als congiunzione angeht, weist sie eher einen parenthetischen Status auf, was orthographisch durch die Kommata angezeigt wird. Um das Stellungsverhalten der congiunzione anche zu illustrieren, führt De Cesare (2004: 193) folgende Beispiele aus der Literatur an: 
(10) Preparati per tempo a una professione; anche, evita la compagnia dei perdigiorno. (Sabatini/Coletti 1997)

,Bereite dich rechtzeitig auf einen Beruf vor; auch, verbringe nicht deine Tage in Gesellschaft der Faulenzer'.

(11) "Ma smettila", disse brutalmente, "ora, anche, mi vuoi fare male" (Moravia)

'»Hör doch auf», sagte er/sie brutal, «jetzt willst du mir auch weh tun»'

(12) "I signori fanno le iniezioni. E lei si è abituata con loro. Ma forse ha un po' di tisi, anche." (Vittorini)

,Die Herren machen die Spritzen. Und sie hat sich an sie gewöhnt. Aber vielleicht hat sie auch Tuberkolose.

Auch in der satzverknüpfenden Funktion ist die additive Grundbedeutung von auch und anche deutlich sichtbar. Sie kommt im Vergleich zur ersten Funktion „Additivität“ auf textueller Ebene zum Tragen, indem auch und anche als Kohäsionsmittel verwendet werden. Dies geht mit einem klaren Unterschied im syntaktischen Status einher: Additives auch/anche ist Teil einer Phrase bzw. eines Satzgliedes, satzverknüpfendes auch/anche ist selbst ein (adverbiales) Satzglied und kann daher allein im Vorfeld stehen bzw. parenthetisch gebraucht werden.

\subsection{Fokusdomäne}

Auch und anche können die Fokusdomäne der Äußerung bilden und damit den Satzakzent tragen. In dieser Funktion wird die Grundbedeutung, i. e. die Operation des Addierens selbst, fokussiert. Dabei stellt der Rest des Satzes informationsstrukturell den Hintergrund dar, wobei die Bezugskonstituente stets ein Topik ist. Die syntaktischen Realisierungsstrategien dieser Funktion unterscheiden sich jedoch in den zwei Sprachen sehr stark. Deutsches auch als Fokusdomäne tritt immer im Mittelfeld auf und ist seinem Bezugsbereich nachgestellt. Ein Beispiel hierfür ist (13) aus Benazzo/Dimroth (2015: 12):

(13) (Paul ist in den Urlaub gefahren.)

$[\text { Johannes }]_{\text {Topik/BB }}$ ist $[\mathbf{A U C H}]_{\text {Fokus }}$ in den Urlaub gefahren.

Dieses Beispiel bildet ein Minimalpaar mit Beispiel (14), in dem auch hingegen die erste Funktion, ,Additivität“, realisiert:

(14) (Paul ist in den Urlaub gefahren.)

Auch [JOHANNES $]_{\text {Fokus/BB }}$ ist in den Urlaub gefahren.

Während der Sachverhalt in (13) und (14) derselbe ist, unterscheiden sich die zwei Sätze im Hinblick auf ihre Fokus-Hintergrund-Gliederung. In beiden Fällen wird zum Ausdruck gebracht, dass Johannes zu der Liste der Personen hinzugefügt werden soll, die im gegebenen Kontext in den Urlaub gefahren sind. Der informationsstrukturelle Status von Johannes ist aber in den zwei Sätzen unterschiedlich: Während in (14) Johannes als Fokus hervorgehoben wird, gilt es in (13) als gegebene Information. Was hier fokussiert wird, ist das Hinzufügen selbst. Dabei kann der Bezugsbereich von auch als Fokusdomäne ein Topik unterschiedlicher Art sein. Handelt es sich um ein Shift-Topik - i. e. nach der Kategorisierung von Frascarelli/Hinterhölzl (2007) eine Konstituente, die ein neues Thema einführt - wie in (13), muss dieses immer realisiert werden. Im Fall eines sogenannten Familiaritätstopiks (cf. Frascarelli/Hinterhölzl 2007) hingegen kann es auch weggelassen werden, wie in (15) aus Reis/Rosengren (1997: 249): 
(15) Ø Hab ich $[\mathbf{A U C H}]_{\text {Fokus }}$ schon erledigt.

In (15) kann fokussiertes auch als Bezugskonstituente nur ein unmittelbar im vorangegangenen Kontext erwähntes Element haben. Dies lässt sich anhand folgenden von uns zur Veranschaulichung konstruierten Beispiels, zu dem auch der Kontext hinzugefügt wird, deutlicher zeigen:

(16) Sprecher A: Im Sommer werde ich Urlaub machen und den Aufsatz fertig schreiben.

Sprecher B: Und wie sieht es mit deinem Buch aus?

A: $\varnothing$ werde ich AUCH fertig schreiben.

Was das Italienische betrifft, ist die Verwendung von anche als Fokusdomäne markiert und nicht uneingeschränkt akzeptierbar. Dies bestätigt auch die Untersuchung von Benazzo/Dimroth (2015: 22) zum L1- und L2-Spracherwerb des Deutschen und des Italienischen. Dabei zeigen sie, dass Sprecher germanischer Sprachen (Deutsch und Niederländisch) dazu tendieren, fokussiertes auch im Mittelfeld zu realisieren. Demgegenüber ziehen Sprecher des Italienischen vor, anche als nicht fokussierte additive Partikel zu verwenden. Auch weist De Cesare (2015: 37f.) darauf hin, dass anche als Fokusdomäne ist ,perceived as less natural [...] and more marked“", wobei sie folgendes Beispiel anführt:

(17) (Luca ha preteso il rimborso del viaggio.)

Gianni $[\mathbf{A N C H E}]_{\text {Fokus }}$ ha preteso il rimborso del viaggio.

(Luca hat die Erstattung der Reisekosten verlangt.) Gianni hat auch die Erstattung der Reisekosten verlangt.‘

Die Markiertheit einer solchen Struktur scheint an der Verletzung des sogenannten ,principle of right scope“ (Andorno 2008) zu liegen. Dieses Prinzip besagt, dass der Bezugsbereich von anche rechts von ihm stehen muss. Eine Bestätigung dieser Erklärung liefert die Präferenz des Italienischen für eine andere Struktur, bei der anche mit einer ihm nachgestellten Bezugskonstituente in Form eines Personalpronomens auftritt, das ein Topik am Satzanfang wiederaufnimmt. Beispiele hierfür sind (18) und (19) respektive aus Kolmer (2012: 191) und De Cesare (2015: 40; cf. auch Benazzo/Dimroth 2015: 22):

(18) [I bambini europei $]_{\mathrm{i}}$ possono anche $\operatorname{loro}_{\mathrm{i}}$ supportare il tour di Nuna 2

Die europäischen Kinder können auch sie unterstützen die Tour von Nuna 2

,Die europäischen Kinder können auch die Nuna2-Tour unterstützen.

(19) $[\text { Stella }]_{i}$ legge anche lei $i_{\text {. }}$

Stella liest auch sie.

,Stella liest auch.

In (18) und (19) realisiert anche zwar die erste Funktion (,Additivität"), indem es vor seinem BB steht und nicht fokussiert ist. Andererseits ist der Bezugsbereich ein Pronomen, das das topikalisierte Element links von anche wiederaufnimmt. Auf diese Weise entspricht die Informationsstruktur dieser Sätze zum Teil der des deutschen auch als Fokusdomäne. Der informationsstrukturelle Status der Partikel selbst bleibt aber selbstverständlich unterschiedlich. Auf jeden Fall unterscheiden sich Satz (18) und (19) informationsstrukturell von denen ohne Pronominalkopie wie (20) und (21):

(20) Anche i bambini europei possono supportare il tour di Nuna2.

,Auch die europäischen Kinder können die Nuna2-Tour unterstützen. ‘ 
(21) Anche Stella legge.

,Auch Stella liest.

\subsection{Modalität}

Eine weitere Funktion, die auch und anche haben können, besteht darin, die semantische Kategorie der Modalität zum Ausdruck zu bringen, das heißt: „,die Stellungnahme des Sprechers zur Geltung des Sachverhalts, auf den sich die Aussage bezieht“ (Bußmann 2002: 438). In dieser Funktion fügen auch und anche nicht ihren BB einer Liste hinzu. Vielmehr beziehen sie sich auf den ganzen Satz, in dem sie auftreten, indem sie diesen mit dem aktuellen Kontext oder mit einer vorangegangenen Äußerung in Verbindung bringen. Durch diese Operation des Verknüpfens ergibt sich eine Sprecherperspektivierung mit unterschiedlichen pragmatischen Effekten. Dabei lassen sich diese auf eine abschwächende bzw. begründende Funktion zurückführen. Das deutsche auch wird in diesem Fall zu der Klasse der Modalpartikeln gerechnet. In der italienischen Forschungsliteratur ist der Terminus Modalpartikeln bzw. particella modale kaum gebräuchlich, taucht aber in der deutsch-italienischen kontrastiven Forschung auf (cf. Burkhardt 1985; Held 1985; Radtke 1985 und Coniglio 2008).

Auch und anche tragen in dieser Funktion nie den Fokusakzent des Satzes und sind stets syntaktisch integriert, i. e. im Deutschen tritt auch im Mittelfeld auf, im Italienischen steht anche zwischen den Elementen des Verbalkomplexes bzw. zwischen Modalverb und lexikalischem Verb oder es folgt unmittelbar auf diese, wobei der parenthetische Gebrauch ausgeschlossen ist.

Im Deutschen tritt auch als Ausdruckmittel der Modalität in folgenden Satzmodi auf:

(i) Aussagesatz

(ii) W-Fragesatz

(iii) W-Exklamativsatz

(iv) Ja-Nein-Fragesatz

(v) Imperativsatz

Beispiele für jede dieser Typologien werden in Thurmair (1989: 155-160) angeführt. In (22) tritt auch in einem Aussagesatz auf (cf. Thurmair 1989: 155):

(22) Ali: Ich hab von dem Text nicht alles verstanden.

Max: Naja, Deutsch ist auch nicht einfach.

Mit auch signalisiert Sprecher Max, dass seine Äußerung im Einklang mit der vorangegangenen Äußerung von Sprecher Ali steht. Durch Inferenz wirkt Max’ Aussage als begründende Instanz zu Alis Feststellung der eigenen Verständnisschwierigkeit. Diese wird somit auch abgeschwächt. In (23) bis (26) wird jeweils ein Beispiel pro Satzmodus angeführt:

(23) Ina: Oh Gott, jetzt hat die schöne Seidenbluse einen Fettfleck!

Mutter: Ja, warum ziehst du auch zum Kochen keine Schürze an? (Thurmair 1989: 159)

(24) Edler von Salchow und Ferdinand von Germitz. Nichtsnutze vom Lande. [...] Wenn sie welche gefunkt kriegten, dann lachten sie: Geschieht uns ganz recht, oh, oh, was sind wir auch für blöde Kerle. (Kempowsky 1982: 236)

(25) Am Beginn eines Urlaubs: Haben wir auch genug Geld dabei? (ibd.: 157) 
(26) Nun iss auch schön! (ibd.: 158)

In allen diesen Beispielen zeigt auch an, dass der Satz, in dem es steht, zum Kontext passt. Dadurch wird die durch den auch-Satz ausgedrückte Sprechhandlung abgeschwächt. Bei (23) und (24) handelt es sich um Vorwürfe, bei (25) und (26) um Aufforderungen.

Was das Italienische betrifft, ist die Verwendung von anche als Modalpartikel nur in Aussagesätzen möglich. In allen anderen Kontexten, in denen auch im Deutschen auftreten kann, ist anche im Italienischen ausgeschlossen (Bidese et al. 2019). Als Beispiel für anche als Modalpartikel führen wir folgenden Beleg aus dem Netz an:

(27) per prova ho messo in bocca una briciola microscopica di tonno sott'olio [...] l'ho sputato schifato [...] troppo forte e salatissimo [...] come anche le olive in salamoia, riesco a mangiarle solo in mezzo all'insalata ma da sole no, troppo salate. Beh è anche normale dopo due mesi e mezzo che ho eliminato il sale.

,als Test habe ich mir einen mikroskopisch kleinen Krümel Thunfisch in Öl in den Mund gesteckt $[\ldots]$ ich habe ihn angeekelt ausgespuckt [...] zu heftig und sehr salzig... ebenso wie eingelegte Oliven, die kann ich nur mit Salat essen, aber nicht alleine, zu salzig. Naja, es ist auch normal nach zweieinhalb Monaten salzfreier Ernährung.' (Forumeintrag: Ben: „Re: nuovo arrivo (sia ringraziato che ci sono arrivato!! An“, 16.9.2010: 11:09 am)

Ähnlich wie auch in Beispiel (22) signalisiert der Schreiber in (27), dass die anche-Äußerung im Einklang mit den bereits gemachten Äußerungen steht. Durch Inferenz wirkt der Satz mit anche als Begründung bzw. Rechtfertigung für das Zuvorgesagte.

In der Literatur wurde beobachtet, dass anche in Kombination mit dem Modalverb potere ('können') in einem Vergangenheitstempus (imperfetto oder condizionale passato) einen deontischen, und somit modalen Wert haben kann (cf. Andorno 2000: 53f., 81):

(28) Potevi anche pensarci! (Andorno 2000: 54)

,Du hättest auch daran denken sollen!‘

Auch in dieser vierten Funktion kommt die additive Grundbedeutung von auch und anche zum Tragen. Sie operiert aber in Zusammenhang mit den Satzmodi, in denen auch und anche auftreten können, und drückt somit den modalen Wert von Epistemizität oder Deontizität aus, der in dem abschwächenden Effekt des Satzes resultiert. In den Aussagesätzen wirken auch und anche auf der epistemischen Ebene der Modalität, indem sie die Sprecherperspektive als Begründung für das Vorhergesagte einführen. In den anderen Satzmodi im Deutschen (Beispiele (23) bis (26)) und in den Aussagesätzen mit dem Modalverb potere im Italienischen operieren auch und anche auf der deontischen Ebene der Modalität. In beiden Fällen schwächen sie den Vorwurf bzw. die Aufforderung ab.

\subsection{Zwischenfazit}

Alle in den Kapiteln 2.1 bis 2.4 dargestellten Eigenschaften von auch und anche lassen sich in folgender Tabelle zusammenfassend veranschaulichen, wobei zunächst die Gemeinsamkeiten der einzelnen vier Funktionen und dann die sprachspezifischen Unterschiede angeführt werden. 


\begin{tabular}{|c|c|c|}
\hline Funktion & auch & anche \\
\hline Additivität & \multicolumn{2}{|c|}{$\begin{array}{l}\text { - Additivität unterhalb der Satzebene } \\
\text { - kein Fokusakzent } \\
\text { - Adjazenz zum BB (Voranstellung präferiert) } \\
\text { - BB mit Fokusakzent }\end{array}$} \\
\hline \multirow[t]{2}{*}{ Satzverknüpfung } & \multicolumn{2}{|c|}{$\begin{array}{l}\text { - Additivität oberhalb der Satzebene bzw. auf textueller Ebene } \\
\text { - kein Fokusakzent } \\
\text { - Adverbialphrase }\end{array}$} \\
\hline & - typischerweise im Vorfeld & - parenthetischer Gebrauch \\
\hline \multirow[t]{2}{*}{ Fokusdomäne } & \multicolumn{2}{|c|}{$\begin{array}{l}\text { - Fokussierung der Additivität (Fokusakzent) } \\
\text { - BB Topik } \\
\text { - Nachstellung zum BB obligatorisch }\end{array}$} \\
\hline & - unmarkiert (typisch) & $\begin{array}{l}\text { - stark markiert } \\
\text { - Pronominalkopie (principle of } \\
\text { right scope) }\end{array}$ \\
\hline \multirow[t]{2}{*}{ Modalität } & \multicolumn{2}{|c|}{$\begin{array}{l}\text { - Additivität auf modaler (epistemischer bzw. deontischer) Ebene } \\
\text { - kein Fokusakzent } \\
\text { - syntaktisch integriert } \\
\text { - Abschwächung }\end{array}$} \\
\hline & - in mehreren Satzmodi & - nur in Aussagesätzen \\
\hline
\end{tabular}

Tabelle 1: Überblick über die vier Funktionen von auch und anche

Die vier Funktionen lassen sich insofern auf einem Kontinuum zunehmender Abstraktion anordnen, als sie sich ausgehend von einer lexikalischen additiven Bedeutung hin zu Modalitätsausdrücken entwickelt haben (cf. Bidese et al. 2019). Am linken Pol des Kontinuums befinden sich auch und anche in der ersten und zweiten Funktion, in der die Additivität auf lexikalischer Ebene zum Tragen kommt, wobei eine Konstituente oder einen ganzen Satz zu einer Liste von vorhandenen Elementen hinzugefügt wird. In der Mitte des Kontinuums liegt die Funktion „Fokusdomäne“. Diese ist im Vergleich zu den ersten zwei insofern abstrakter, als nicht das addierte Element, sondern die Operation des Addierens selbst fokussiert wird. Damit äußert sich die Additivität nicht auf rein lexikalischer, sondern vielmehr auf informationsstruktureller Ebene. Am rechten Pol des Kontinuums haben wir schließlich die abstraktere Funktion der Modalität. Hierbei betrifft die Operation des Addierens Propositionen bzw. Sprechakte, in denen eine Sprecherperspektivierung zum Tragen kommt. Das Resultat dieser Form der Additivität ist eine Abschwächung auf epistemischer oder deontischer Ebene. In dieser Funktion operieren auch und anche auf syntaktischer Ebene, indem sie als Köpfe eigener Modalitätsprojektionen fungieren (cf. Coniglio 2011) und stellen somit nach Abrahams (2020) Klassifikation grammatische Modalitätsausdrücke mit doppelter Deixis dar: (i) sie verweisen auf die Quelle der Proposition und (ii) auf die Bewertung seitens des Sprechers bzw. - im Falle der Modalpartikel - auch in Bezug auf die Perspektive des Hörers. 


\begin{tabular}{|l|c|l|c|c|}
\hline & Modalitätsausdrücke & \multicolumn{1}{|c|}{ Beispiel } & Quelle & Bewertung \\
\hline lexikalisch & offensichtlich & $\begin{array}{l}\text { Haider ist } \text { offensichtlich } \\
\text { betrunken gewesen }\end{array}$ & + & - \\
\cline { 2 - 5 } & $\begin{array}{l}\text { wahrscheinlich/ } \\
\text { vermutlich }\end{array}$ & $\begin{array}{l}\text { Haider ist } \text { wahrscheinlich } \\
\text { /vermutlich betrunken } \\
\text { gewesen }\end{array}$ & - & + \\
\hline $\begin{array}{l}\text { grammati- } \\
\text { kalisch }\end{array}$ & sollen & $\begin{array}{l}\text { Haider } \text { soll betrunken ge- } \\
\text { wesen sein }\end{array}$ & + & + \\
\cline { 2 - 5 } & $j a$ & $\begin{array}{l}\text { Heider ist ja betrunken } \\
\text { gewesen }\end{array}$ & + & ++ \\
\hline
\end{tabular}

Tabelle 2: Klassifikation der Modalitätsausdrücke in Anlehnung an Abraham (2020: 44f.)

Abraham (2020) unterscheidet zwei Haupttypen von Modalitätsausdrücken: lexikalischen und grammatikalischen. Erstere sind modale Adverbien wie offensichtlich, vermutlich und wahrscheinlich, bei denen entweder die Quelle der Proposition (offensichtlich) oder deren Bewertung seitens des Sprechers (wahrscheinlich, vermutlich) kodiert wird (siehe das + in der obigen Tabelle). Letztere sind Modalverben und Modalpartikeln wie beispielsweise das epistemische Modalverb sollen oder die Modalpartikel ja; bei ihnen werden beide Deixis kodiert. Darüber hinaus wird bei den Modalpartikeln auch die Perspektive des Hörers in die Bewertung des Sprechers miteinbezogen.

Abrahams Generalisierungen sind auf der Basis der deutschen Modalitätsausdrücke formuliert, sie lassen sich jedoch zum großen Teil auf das Italienische übertragen. In diesem Sinne stellen modale auch und anche die letzte Stufe eines Sprachwandelprozesses dar, dessen Bestimmung in der Literatur noch zur Debatte steht, insofern er entweder als Grammatikalisierung oder Pragmatikalisierung definiert wird (cf. Diewald 2011).

\section{Empirische Validierung}

\subsection{Deutsches auch}

Es gilt nun die unter Kapitel 2 aus der Literatur hergeleiteten Funktionen von auch und dessen Realisierungsweisen anhand authentischer Daten zu überprüfen. Hierzu werden nähesprachliche Daten aus einem Unterhaltungsgespräch unter Freunden aus der Datenbank FOLK (Forschungs- und Lehrkorpus Gesprochenes Deutsch) des Instituts für deutsche Sprache in Mannheim herangezogen. Am Gespräch (Kennzeichen „FOLK_00066“) nehmen vier Freunde teil, die zusammen im Garten von einem von ihnen sitzen und Bier trinken. Dabei unterhalten sie sich über verschiedene Themen, z. B. eine Party, an der einige von ihnen am vorigen Tag teilgenommen haben, Musikfestivals, die Scheidung eines der Teilnehmer, Fahrräder, Preise und Typen von Ape Rollern. Sie sprechen die deutsche regionale Varietät aus Freiburg, i. e. ihr Deutsch weist einen alemannischen Anschlag auf. Aus dem Gespräch wurden die ersten 110 Vorkommen von auch bzw. au herausgefiltert: Von den 110 Belegen sind 68 auch und 42 au. Auch und au scheinen gleichwertig zu sein bzw. es scheint kein Verwendungsunterschied zwischen den beiden Formen zu bestehen. Die 110 Vorkommen werden im Hinblick auf ihre 
Verteilung auf die vier oben dargestellten Funktionen analysiert. Zunächst ist es hilfreich, sich die syntaktische Position der Belege vor Augen zu führen:

\begin{tabular}{|c|c|}
\hline Anzahl der Vorkommen & Syntaktische Position \\
\hline 3 & auch wenn \\
\hline 3 & Ellipse \\
\hline 2 & Nachfeld \\
\hline 102 & Mittelfeld \\
\hline
\end{tabular}

Tabelle 3: syntaktische Position der auch-Vorkommen

An dieser Übersicht lässt sich zunächst ablesen, dass im Korpus Vorkommen der Partikel auch, die zusammen mit ihrem BB im Vorfeld steht, nicht belegt sind. Das heißt: Beispiele, die in der Grammatikschreibung als prototypisch für die Verwendung von auch gelten - wie das oben bereits angeführte Beispiel (4a), hier als (29) wiederholt, oder Beispiel (30), das aus der DudenGrammatik (2016: 602) stammt - kommen im Korpus nicht vor:

(29) Auch [Peter]BB hat bei Lucie angerufen. (Pasch et al. 2003: 577)

(30) Auch [die Lehrerin]BB spielte mit (andere auch). (Duden 2016: 602)

Auch Belege für auch alleine im Vorfeld (in der Funktion (ii) „Satzverknüpfung“) sind nicht vorhanden. Hierzu könnte die Hypothese aufgestellt werden, dass auch mit dem BB im Vorfeld und auch alleine im Vorfeld womöglich nicht in nähesprachlichen Daten, wie denen des Korpus, sondern eher in distanzsprachlichen auftreten. Eine Stichprobe von 100 auch-Belegen aus DeReKo aus den Ausgaben der Süddeutschen Zeitung des Jahres 2018 hat ergeben, dass auch mit dem eigenen BB im Vorfeld 13 mal belegt ist und dass auch alleine im Vorfeld 2 mal vorkommt. Dies scheint darauf hinzudeuten, dass diese zwei Positionen von auch vorzugsweise in schriftlichen bzw. distanzsprachlichen Texten auftreten, wobei dies anhand einer eingehenden Untersuchung verifiziert werden sollte.

Kommen wir nun zu den syntaktischen Positionen von auch, die im Korpus belegt sind. In drei Fällen tritt auch zusammen mit wenn auf und leitet einen konzessiven Nebensatz ein. In weiteren drei Fällen ist es Teil einer verblosen Struktur bzw. Ellipse, wobei sich die Funktion von auch jeweils anhand des Kontextes desambiguieren und funktional nach unserer Kategorisierung klassifizieren lässt, wobei Graubereiche bestehen können. Schauen wir hierzu folgendes Beispiel an:

(31) FOLK_00066 (Transkriptteil 2) $)^{5}$

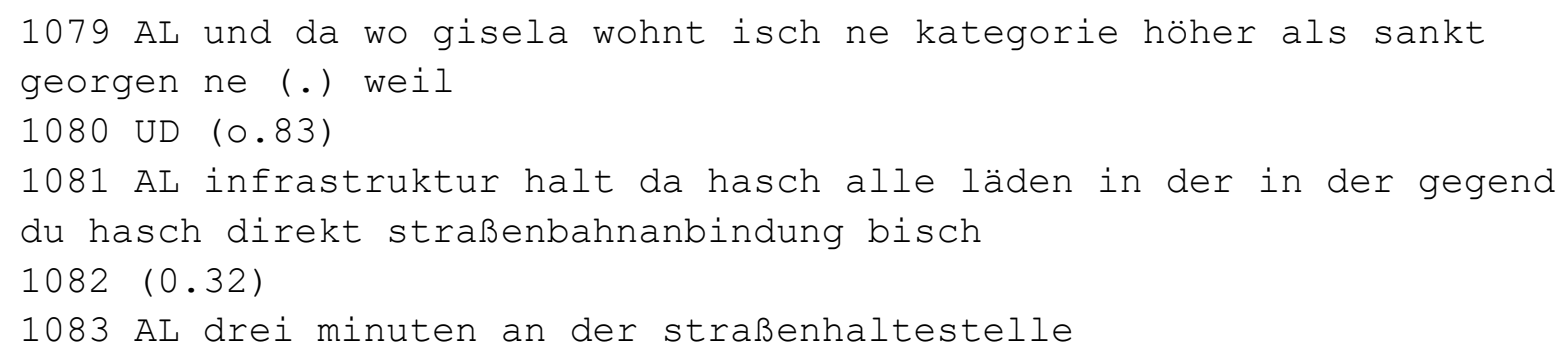

5 Die gesprochen-sprachlichen Beispiele werden nach den Transkriptionskonventionen von GAT2 (Selting et al. 2009) wiedergegeben. 
$1084 \quad(0.46)$

1085 AL schule hasch in der in der gegend kindergärten auch da

In dieser Passage zählt Sprecher AL mehrere Vorteile eines Viertels auf, in dem eine Bekannte von ihm wohnt. Am Ende der Aufzählung fügt er einen letzten Vorteil hinzu durch die elliptische Äußerung Kindergärten auch da. Diese lässt sich als Kindergärten sind auch da explizieren, wobei auch in dieser Position (Mittelfeld) u. E. auf den ersten Blick als Ausdrucksmittel der Funktion Fokusdomäne interpretiert werden kann, wie in (32):

(32) $[\text { Kindergärten }]_{\mathrm{BB} / \text { Topik }}$ sind $[\mathbf{A U C H}]_{\text {Fokus }}$ da.

Bei mehrmaligem Anhören dieser Stelle im Gespräch lässt sich allerdings kein Fokusakzent in Korrespondenz von auch erkennen. Ohne Fokusakzent kann auch als Ausdruckmittel der Satzverknüpfung gedeutet werden, wobei der Kontext eher eine Interpretation als Fokusdomäne nahelegt. Dies stellt also einen Zweifelsfall dar, bei dem die Zuordnung der Funktion von auch nicht ganz eindeutig ist.

Zwei Belege im Korpus weisen auch im Nachfeld zusammen mit einer Nominalphrase auf, die durch einen Relativsatz modifiziert wird. Dabei handelt es sich in beiden Fällen um auch in der ersten Funktion (,Additivität“), bei der die Partikel ein Element zu einer Liste hinzufügt. Im Folgenden wird einer der beiden Fälle angeführt:

(33) FOLK_00066 (Transkriptteil 2)

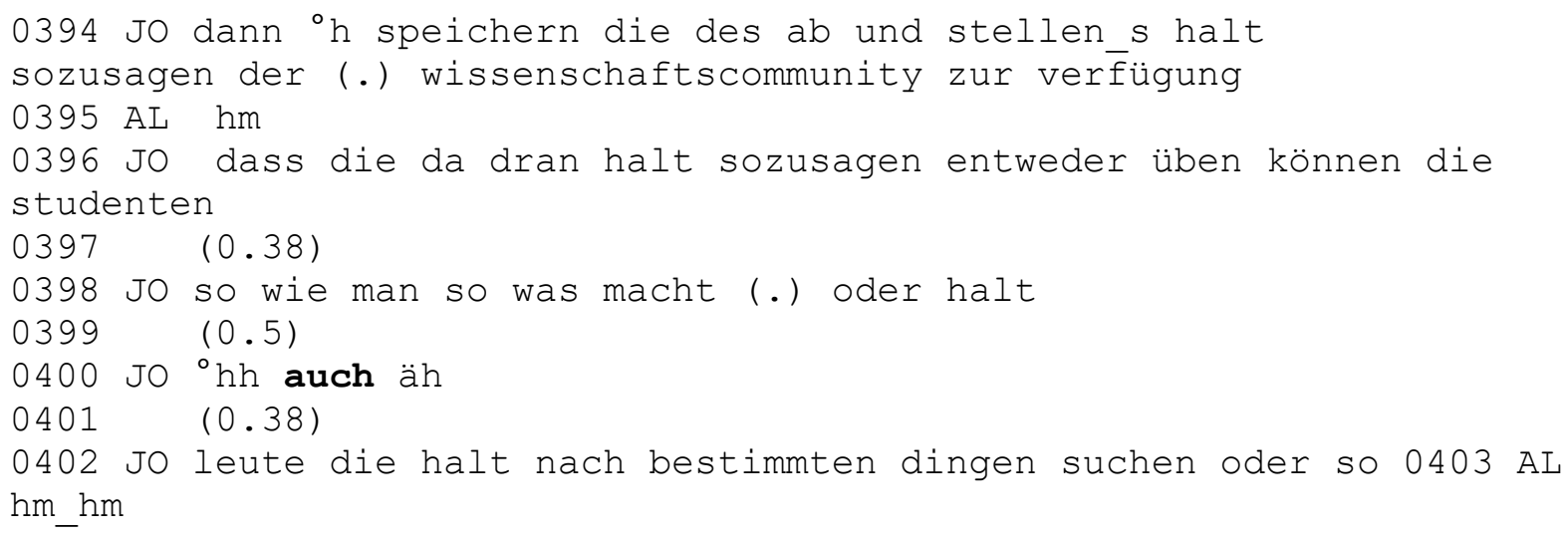

In diesem Auszug erklärt Sprecher JO Sprecher AL, der gerade angekommen ist, dass das Gespräch zu wissenschaftlichen Zwecken aufgezeichnet wird und die Daten dann auch in der Lehre eingesetzt werden können. Der BB von auch ist hier Leute, die halt nach bestimmten Dingen suchen oder so, wobei auch und sein BB im Nachfeld des Konsekutivsatzes dass die da dran halt sozusagen entweder üben können, wo auch die Studenten und so wie man so was macht stehen. Durch auch wird hier der BB Leute, die halt nach bestimmten Dingen suchen oder so zu der Liste der Leute hinzugefügt, die die Daten verwenden können. Zur Liste gehören die bereits erwähnten Studenten und die Wissenschaftscommunity.

In 102 Fällen von 110 - also im Großteil der Korpusbelege - tritt auch im Mittelfeld auf. In dieser Position kann die Funktion von auch nicht automatisch ermittelt werden bzw. kann ambig sein. Die Ambiguität betrifft die Funktionen (i) („,Additivität“), (ii) (,Satzverknüpfung“) und (iv) („Modalität“"). Demgegenüber sind jedoch aufgrund der Anwesenheit des Fokusakzents die Belege für die Funktion (iii), Fokusdomäne, eindeutig zu erkennen. Diese sind 
insgesamt 32. Zunächst gehen wir auf auch als Fokusdomäne ein. Anschließend besprechen wir die Zweifelsfälle.

Ein Beispiel aus dem Korpus für auch als Fokusdomäne ist (33). In dieser Passage erzählt Teilnehmer PA, was es auf der Party vom Vortag zu essen gab. Dabei nennt er u. a. auch Pute. Daraufhin sagt JO, dass er Pute nicht mehr essen kann, seitdem er gesehen hat, wie Puten gezüchtet werden. PA reagiert auf JOs Anmerkung mit der ironischen Äußerung in Zeile 0092, in der au auftritt:

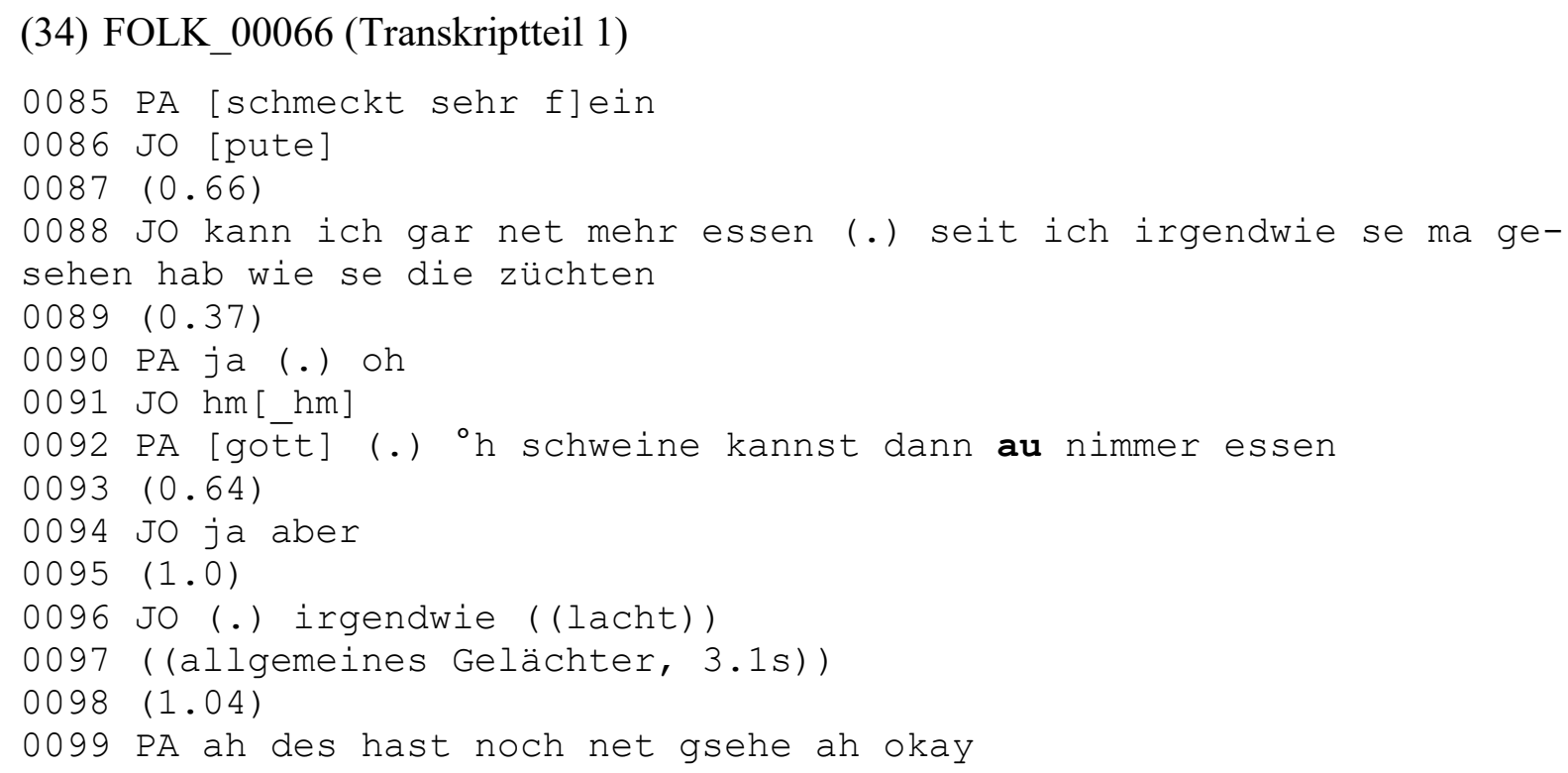

In PAs Äußerung in Zeile 0092 trägt $a u$ den Fokusakzent, wobei die Konstituente Schweine im Vorfeld den BB und zugleich das Topik bildet:

(35) $[\text { Schweine }]_{\mathrm{BB} / \mathrm{Topik}}$ kannst dann $[\mathbf{A U}]_{\text {Fokus }}$ nimmer essen.

Von den 32 Vorkommen für auch (bzw. au) als Fokusdomäne sind 19 analog zu (34)/(35), indem der BB im Vorfeld und fokussiertes auch im Mittelfeld steht. In den restlichen 13 Vorkommen steht demgegenüber der BB nicht im Vorfeld, sondern im Mittelfeld (links von auch). Ein Beispiel hierfür ist (36):

(36) FOLK_00066 (Transkriptteil 2)

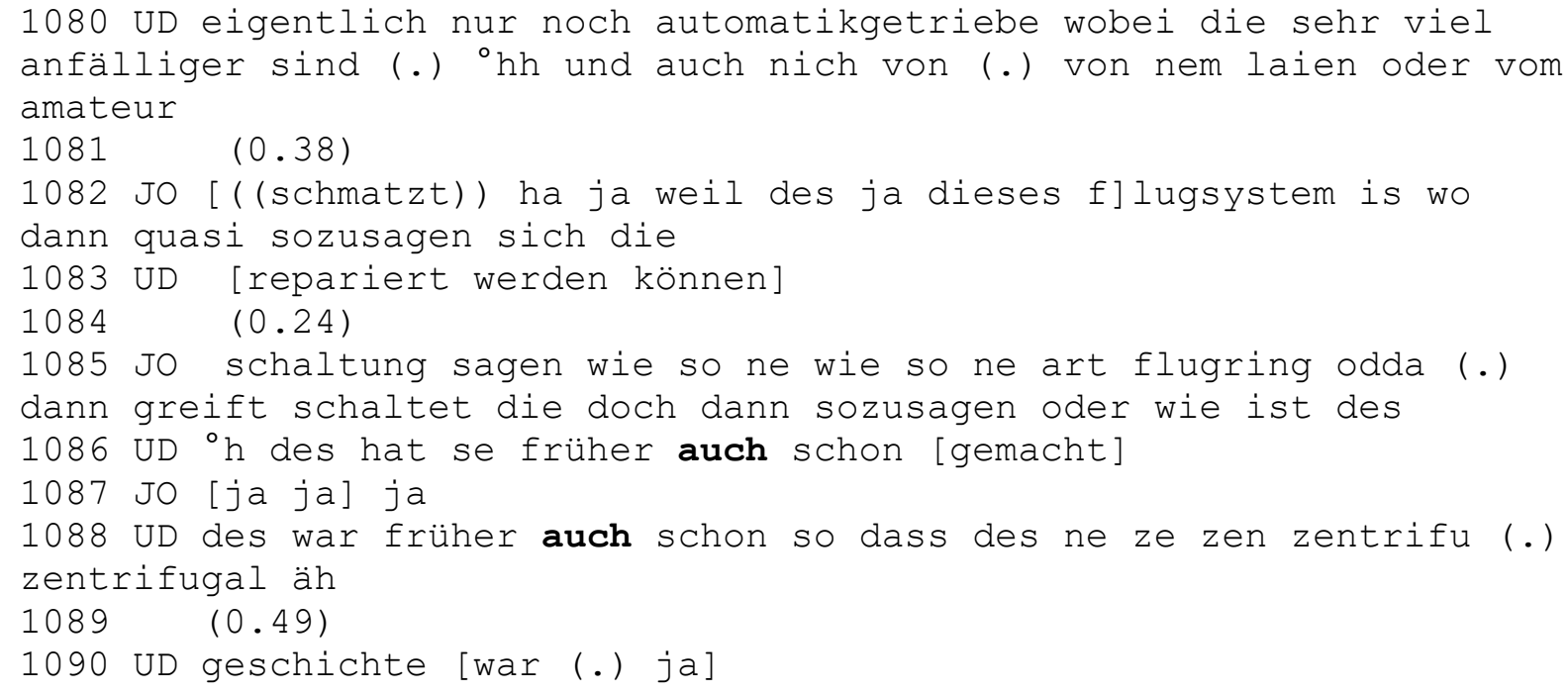


In diesem Auszug sprechen Teilnehmer JO und UD über alte und neue Ape Roller und die Unterschiede zwischen den Schaltgetrieben der alten Ape Roller und den Automatikgetrieben der neuen. Am Anfang dieses Auszugs (Zeile 1080) sagt UD, dass die heutigen Ape Roller nur noch ein Automatikgetriebe haben, und dass dieses von Amateuren schwer zu reparieren ist. Daraufhin wirft JO einen Kommentar ein, indem er versucht zu erklären, warum sich das Automatikgetriebe nur schwer reparieren lässt (wegen des Flugrings). Auf diesen Kommentar reagiert ab Zeile 1986 Sprecher UD mit einer Korrektur von JOs Erklärungsversuch. Hierbei realisiert er zwei ähnliche Äußerungen mit fokussiertem auch in Zeile 1086 und 1088:

(37) Des hat se $[\text { früher }]_{\mathrm{BB} / \text { Topik }}[\mathbf{A U C H}]_{\text {Fokus }}$ schon gemacht.

(38) Des war $[\text { früher }]_{\mathrm{BB} / \text { Topik }}[\mathbf{A U C H}]_{\text {Fokus }}$ schon so.

In beiden Fällen ist der BB von fokussiertem auch das Adverbiale früher, das mit "jetzt" bzw. mit den alte Ape Rollern mit Schaltgetriebe kontrastiert wird. Hier lässt sich also feststellen, dass der BB von auch als Fokusdomäne im Mittelfeld stehen kann, wobei hierauf in der Literatur unseres Wissens bisher nicht hingewiesen wurde. Ein weiteres Beispiel hierfür ist (38):

(39) FOLK_00066 (Transkriptteil 2)

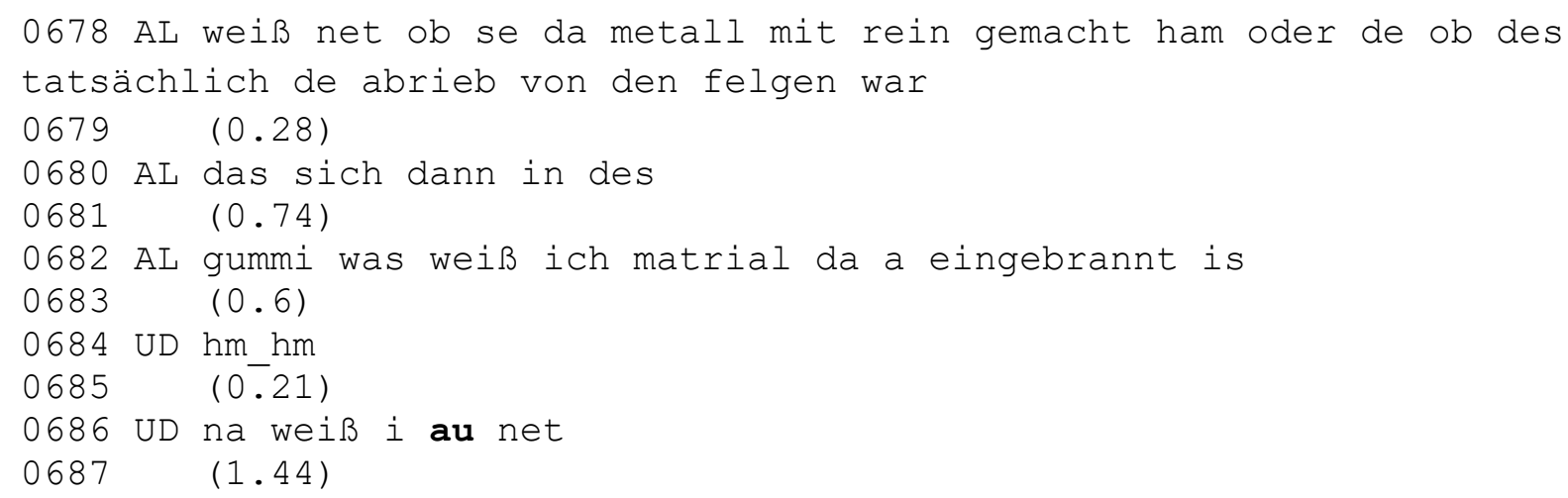

In diesem Auszug ist von Fahrradbremsen die Rede. AL hat erzählt, dass seine Fahrradbremsen abgenutzt sind und fragt sich warum, ohne eine Antwort dazu zu haben (Zeile 0678-0682). Daraufhin signalisiert UD auch seine Ratlosigkeit durch die Äußerung in Zeile 0686, die ein fokussiertes auch aufweist:

(40) Na, weiß $[\mathrm{i}]_{\mathrm{BB} / \text { Topik }}[\mathbf{A U}]_{\text {Fokus }}$ net.

Der BB von $a u$ ist $i$ (ich). UD möchte nämlich sagen, dass er genauso wie AL nicht weiß, wie solche Bremsen funktionieren bzw. wie sie sich abnutzen. Auch in diesem Fall steht der BB links von au innerhalb des Mittelfeldes.

Während von den 110 Vorkommen von auch in FOLK_00066 32 als Fokusdomäne fungieren, ergibt eine Stichprobe aus dem Jahrgang 2018 der Süddeutschen Zeitung in DeReKo nur 3 auch-Vorkommen als Fokusdomäne von 100 Belegen. Dies könnte darauf hindeuten, dass diese Funktion von auch eher in interaktionalen Kontexten auftritt und weniger in distanzsprachlichen Texten (Pressetexten).

Kommen wir nun zu den Zweifelsfällen. In (41) könnte auch zur Funktion „Satzverknüpfung“ oder zu der der „Modalität“ zugeschlagen werden. In dieser Passage bringen JO und UD abwechselnd Argumente dafür, dass Kleinhandwerker Ape Roller statt größerer Fahrzeuge verwenden sollten. 
(41) FOLK_00066 (1 Transkriptteil/2 Transkriptteil)

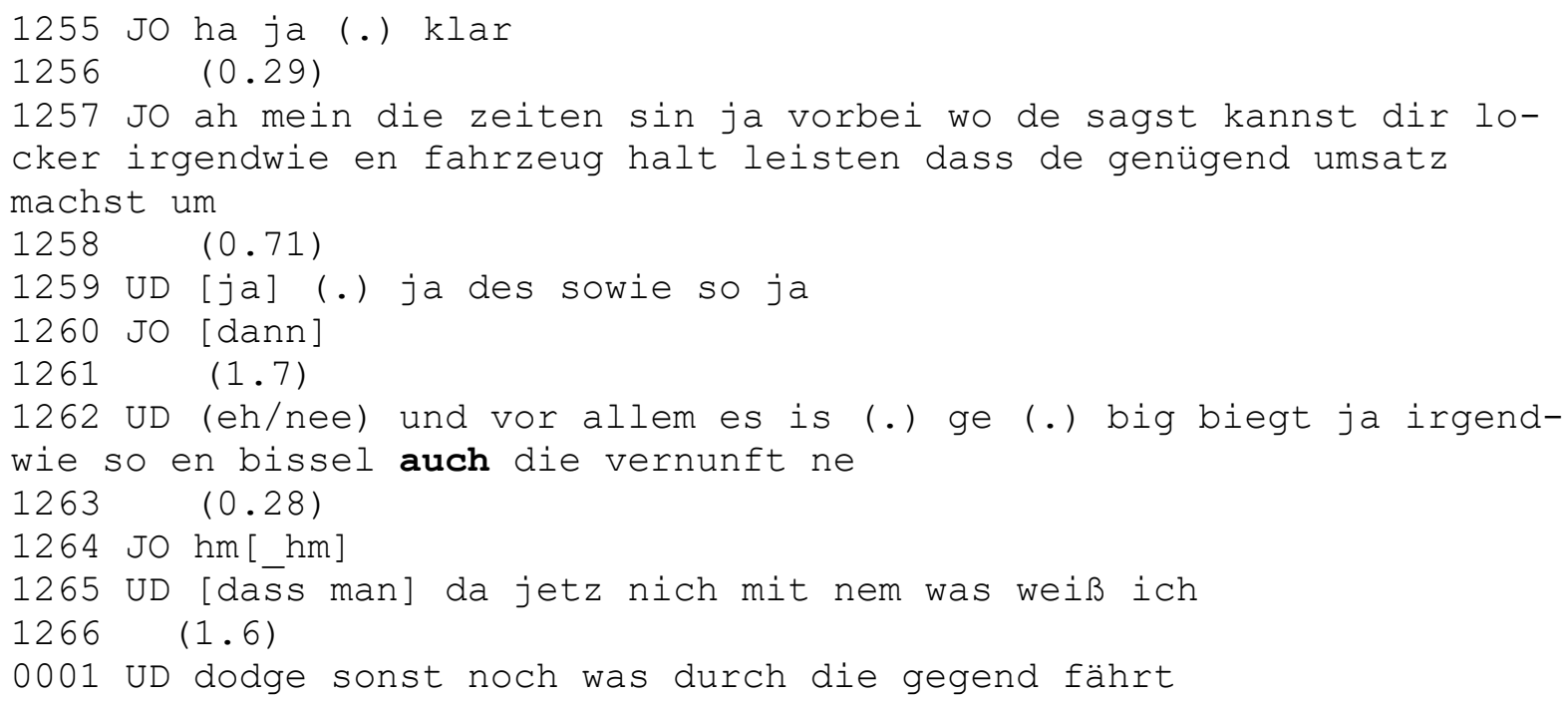

Auch befindet sich hier in folgendem Satz:

(42) Es biegt ja irgendwie so ein bissel auch die Vernunft ne? Dass man da jetz nich mit nem was weiß ich Dodge sonst noch was durch die Gegend fährt.

Zum einen könnte die Partikel auch den Satz von UD, in dem sie auftritt, mit dem von JO in den Zeilen 1255 bis 1260 nicht fertig formulierten Argument verknüpfen, nach dem sich kleine Handwerker heutzutage nicht mehr ein normales Fahrzeug leisten können. Nach dieser Interpretation fungiert hier auch als Satzverknüpfer, wobei die Verknüpfung über die Grenzen eines einzelnen Redebeitrags hinausreicht und zwei Redebeiträge von zwei verschiedenen Teilnehmern miteinander verbindet. Zum anderen ließe sich auch der Funktion „Modalität“ zuschlagen. Nach der modalen Interpretation zeigt Teilnehmer UD durch auch an, dass aus seiner subjektiven Perspektive seinen Kommentar im Einklang mit dem vorherigen seines Gesprächspartners JO (Zeilen 1255 bis 1260) steht.

In (43) liegt ein weiterer Zweifelsfall vor. Die Passage stellt den Beginn einer neuen Gesprächsphase dar, die nach einer langen Pause (Zeile 0996) einsetzt und durch UD initiiert wird.

(43) FOLK_00066 (1 Transkriptteil)

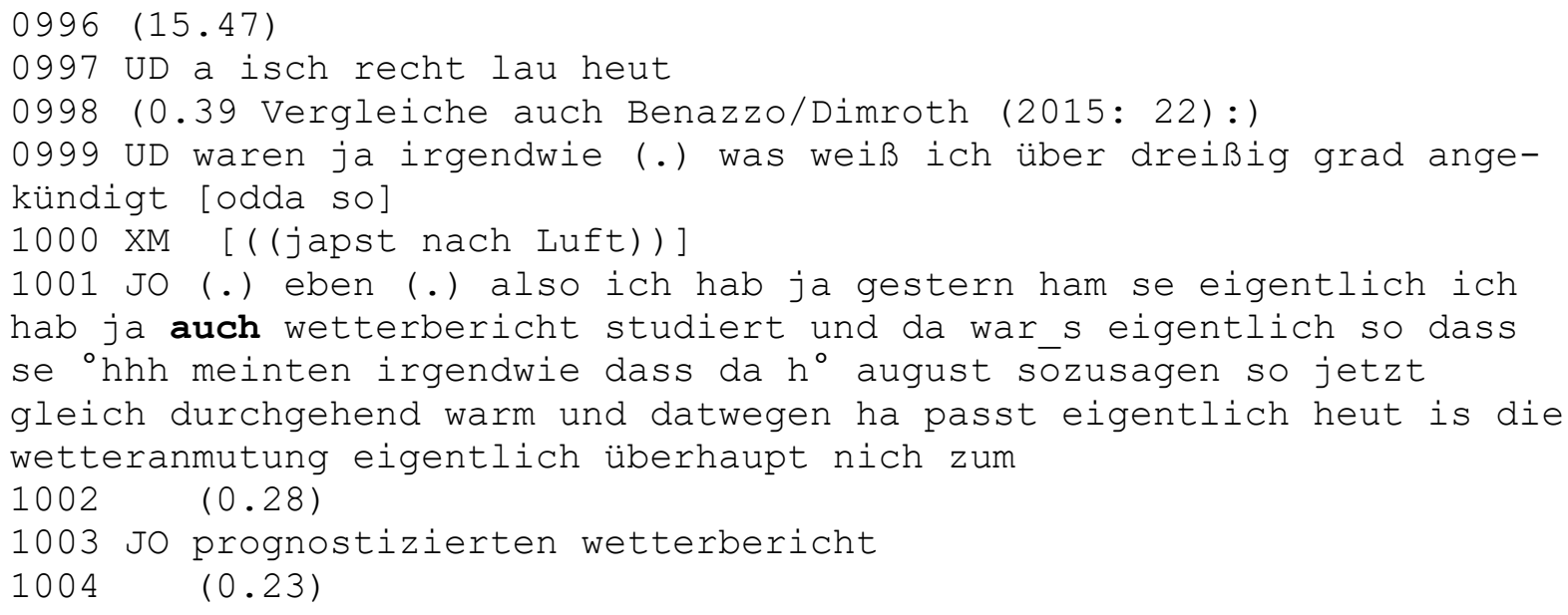


1005 UD ja

$1006 \quad(1.25)$

1007 UD fürcht ich mich ja schon en bisschen vor italien [...]

Im Hinblick auf den Kontext bzw. die Redebeiträge unmittelbar vor der auch-Äußerung lässt sich auch der Funktion „Fokusdomäne“, der der „Modalität“ oder der der „Satzverknüpfung“ zuordnen. Durch auch könnte Sprecher JO anzeigen, dass er genauso wie UD, ,den Wetterbericht studiert hat". An dem, was UD in Zeile 0999 sagt, könne nämlich geschlossen werden, dass er sich über das Wetter informiert hat. Nach dieser Interpretation drückt also auch aus, dass JO zusammen mit UD zu denen gehört, die sich über das Wetter informiert haben. Der BB von auch wäre nach dieser Interpretation die Konstituente ich im Vorfeld des Satzes:

(43) $[\mathrm{Ich}]_{\mathrm{BB} / \text { Topik }}$ hab ja $[\mathbf{A U C H}]_{\text {Fokus }}$ Wetterbericht studiert.

Bei dieser Interpretation müsste auch fokussiert sein, weil es auf seinen BB folgt. Diese Interpretation ist aber problematisch, weil in Korrespondenz von auch kein deutlicher Akzent zu hören ist. Akzentuiert bzw. fokussiert ist vielmehr Wetterbericht, wobei der Akzent allerdings schwach ist und die gesamte Äußerung intonatorisch relativ flach realisiert wird. Wenn man annimmt, dass auch nicht fokussiert ist, dann muss es der Funktion Modalität zugerechnet werden. Nach dieser zweiten Interpretation bringt auch zum Ausdruck, dass JOs Äußerung mit UDs vorangegangenem Redebeitrag im Einklang steht und dass beide Redebeiträge im Hinblick auf die Sprechereinstellung also gleichlaufend sind. Darüber hinaus könnte man auch die Hypothese aufstellen, dass an dieser Stelle auch satz- bzw. redebeitragsverknüpfend wirkt und somit die Bedeutung ,außerdem“ trägt. Dieser Beleg zeigt unseres Erachtens besonders deutlich, dass sich auch nicht immer eindeutig klassifizieren lässt, wobei mehrere Funktionen ,,mitschwingen“.

\subsection{Italienisches anche}

Zur Validierung unserer Analyse von anche haben wir 100 Belege aus dem Korpus Lessico di frequenza dell'italiano parlato (LIP) extrahiert. Diese stammen aus Gesprächen, die in LIP der Typologie A, i. e. informeller Face-to-Face Kommunikation mit ungesteuertem Sprecherwechsel, zugeordnet sind. Die Sprecher stammen aus den vier größten italienischen Städten (Mailand, Florenz, Rom und Neapel). Anders als im Deutschen, in dem auch typischerweise im Mittelfeld auftritt und überwiegend nur mit Rückgriff auf den Kontext funktional bestimmt werden muss, wird italienisches anche im Großteil der Belege (69/100) als additive Partikel verwendet und tritt unmittelbar links des eigenen Bezugsbereichs auf. Dabei kann anche verschiedene syntaktische Positionen einnehmen, die nicht mit den Mitteln der Satzfelderanalyse erfassbar sind. Zum Beispiel tritt anche im Auszug (44) unmittelbar vor seinem Bezugsbereich il sabato auf:

(44) ${ }^{6}($ LIP, Florenz, Typ A, Gespräch 2)

B: ma la CIT è aperta anche [il sabato $]_{\mathrm{BB}}$ ?

A: aperta sempre

\footnotetext{
${ }^{6}$ Die Auszüge aus dem LIP-Korpus werden nach den im Korpus verwendeten Transkriptionskonventionen wiedergegeben.
} 
'B: aber hat die CIT auch am Samstag auf?

A: (die hat) immer auf.'

In (45) ist anche direkt dem Bezugsbereich sua mamma vorangestellt:

(45) (LIP, Rom, Typ A, Gespräch 1)

B: no Claudio di dov'è? Dov'è nato non si sa?

A: a Forlì

$[\ldots]$ A: anche [sua mamma $]_{\mathrm{BB}}$ è di Forlì?

'B: ne, Claudio, woher kommt er denn? Weiß man denn nicht, wo er geboren wurde?

A: in Forlì

[...] A: Stammt seine Mutter auch aus Forli?'

Schließlich tritt anche in (46) - analog zu (44) und (45) - unmittelbar vor dem Bezugsbereich noi auf:

(46) (LIP, Florenz, Typ A, Gespräch 1)

$\mathrm{B}$ : aspetta che mi_se mi dai $<$ ? > lì raccolgo l'aglio

$\mathrm{C}$ : ma l'aglio lo vogliamo anche $[\text { noi }]_{\mathrm{BB}}$

B: no la Giovanna ha detto che non gli piace

'B: Warte mal, wenn du mir $<$ ? > gibst, tue ich den Knoblauch weg.

C: aber den Knoblauch, den wollen wir auch.

B: ne, die Giovanna hat doch gesagt, dass sie keinen Knoblauch mag.'

In 16 Belegen realisiert anche eine satzverknüpfende Funktion, wie in (47):

(47) (LIP, Florenz, Typ A, Gespräch 1)

B: ho fatto colazione mi son messa a lavorare tre ore sono andata a far la spesa ho mangiato poi c'avevo anche sonno

,Ich habe gefrühstückt, hab drei Stunden gearbeitet, bin einkaufen gegangen, habe gegessen, dann war ich auch müde.

Unter den 16 Belegen mit satzverknüpfender Funktion sind auch welche (9), die einen Nebensatz einleiten und zwar in Kombination mit se bzw. perché, wie in (48):

(48) (LIP, Neapel, Typ A, Gespräch 3)

B: siamo rimasti tutti molto ben impressionati veramente molto oltre per diciamo la magnificenza esteriore anche perché era simpatico

,Wir waren alle echt beeindruckt, \# wirklich sehr wegen der, sagen wir, äußerlichen Erscheinung auch weil er sympathisch war'

Anders als bei den schriftlichen Belegen aus literarischen Texten, die wir oben (siehe (10) bis (12)) angeführt haben, zeigen die Beispiele aus der gesprochenen Sprache keine parenthetische Verwendung, sondern sie tauchen nach dem finiten Verb (Beispiel (47)) bzw. dem verbalen Komplex oder unmittelbar vor dem Nebensatzeinleiter (Beispiel (48)) auf. Möglicherweise ist der parenthetische Gebrauch textsortenabhängig bzw. typisch für literarische Quellen.

In einem einzigen Fall scheint anche als Fokusdomäne aufzutreten. Da aber das LIP keine Audiodateien zur Verfügung stellt, konnten wir nicht prüfen, ob anche tatsächlich den Fokusakzent trägt. Der Kontext scheint nahezulegen, dass sich anche auf das Subjekt il medico della mutua etc [...] bezieht, indem es signalisiert, dass il medico della mutua - zusammen mit dem 
bereits genannten Bürgermeister - der Liste der Amtsträger, die ihre Amtspflichten nicht erfüllen, hinzuzufügen ist.

(49) (LIP, Rom, Typ A, Gespräch 1)

B: il sindaco che deve rilasciare la concessione edilizia \# e non lo fa omette un atto del suo ufficio [...] [il medico della mutua che non corre che fa che sta facendo la guardia medica e non corre eh a visitare un malato che invece lo chiama perché è grave $]_{\mathrm{BB}}$ commette anche il reato di omissione di atti d'ufficio [...]

'Ein Bürgermeister, der eine Baugenehmigung erteilen muss und es nicht tut, unterlässt eine Amtshandlung [...] ein Arzt, der in Bereitschaftsdienst ist und nicht rausfährt, um einen Kranken zu besuchen, der ihn erfordert hatte, weil seine Lage ernst ist, begeht auch die Straftat der Unterlassung der Amtshandlung'

Schließlich tritt anche vereinzelt (6/100) mit modaler Funktion auf. Ein Beispiel hierfür ist (50):

(50) (LIP, Florenz, Typ A, Gespräch 1)

D: io ho mangiato alle dodici e mezza oggi

$[\ldots]$

$\mathrm{B}:<$ ?? $>$ una delle poche volte che tu ha' mangiato a quell'ora

[...]

D: perché stamattina mi sono svegliata alle otto \# alle sette

B: ah quindi

D: no scusa alle sette certo perché se ti svegli alle dieci e fai

colazione alle dieci e mezza puoi mangiare anche alle due

'D: Ich habe um halb zwölf gegessen heute

$[\ldots]$

B: $<? ?>$ da isst du sonst nie zu dieser Uhrzeit

[...]

D: ja weil heute Morgen bin ich um acht aufgewacht \# um sieben

B: Ach so, also

D: ne, entschuldige, um sieben, klar, weil wenn du um zehn aufwachst und um halb elf frühstückst, kannst du auch um zwei essen

\subsection{Zusammenfassung der empirischen Validierung}

Die empirische Analyse hat gezeigt, dass sich authentische Daten sowohl des Deutschen als auch des Italienischen anhand der in der Literatur besprochenen und von uns systematisierten vier Funktionen klassifizieren lassen. Dabei hat sich ergeben, dass in den beiden Sprachen die vier Funktionen eine unterschiedliche Gewichtung haben. So tauchen 102 von 110 Belegen von auch im Mittelfeld auf. Im Gegensatz zum Vor- und Nachfeld lässt diese Position alle Funktionen von auch zu, die daher mit Rückgriff auf den Kontext desambiguiert werden müssen. Im Falle vom fokussierten auch ist die Zuordnung zur Funktion der Fokusdomäne durch die Prosodie bzw. den Akzent auf auch eindeutig. Demgegenüber ist diese Funktion in den Daten zu anche kaum vertreten. Vielmehr tendiert anche generell dazu, unmittelbar vor dem eigenen Bezugsbereich realisiert zu werden, was der additiven Funktion entspricht.

\section{Schlussfolgerungen}

Ausgangspunkt der vorliegenden Studie war die Feststellung, dass deutsches auch und italienisches anche trotz der geteilten additiven Semantik in der jeweiligen Sprache unterschiedlich 
verwendet werden. Um einen Sprachvergleich durchzuführen, sind wir funktionsgeleitet vorgegangen. Dabei haben wir zunächst aus der Literatur zu beiden Partikeln vier gemeinsame Funktionen herausgearbeitet, die wir „Additivität“, „Satzverknüpfung“, „Fokusdomäne“ und „Modalität“ genannt haben. Wir haben außerdem gezeigt, dass diese sich in ihren Realisierungsformen im Deutschen und Italienischen unterscheiden und auf einem Kontinuum zunehmender Abstraktion anordnen lassen. Im Anschluss daran haben wir die aus der Literatur basierende Klassifikation der vier Funktionen anhand deutscher und italienischer gesprochensprachlicher Daten überprüft. Insgesamt hat sich ergeben, dass die aus der Literatur hergeleiteten Verwendungsweisen von auch und anche zur Analyse authentischer Daten tauglich sind, wobei Tendenzen im Sprachgebrauch aufgedeckt wurden, die in der Grammatikschreibung in der Regel nicht berücksichtigt werden. Die Ergebnisse der empirischen Validierung für auch und anche lassen sich in folgenden Punkten zusammenfassen:

(i) Die in der Grammatikschreibung als prototypisch betrachtete Verwendung von auch als additive Partikel (mit der gängigen Bezeichnung „Fokuspartikel“) tritt im untersuchten Gespräch zwar auf, aber durchgehend im Mittelfeld und nie im Vorfeld auf. Im Gegensatz dazu orientiert sich die Position von anche mit additiver Funktion an den Bezugsbereich.

(ii) Auch allein im Vorfeld (in der Funktion „Satzverknüpfung”) ist in unseren gesprochensprachlichen Daten nicht belegt. Dies deutet darauf hin, dass diese Verwendung eher schrift- bzw. distanzsprachlich ist. Was anche mit satzverknüpfender Funktion betrifft, tritt es anders als bei den in der Literatur besprochenen Beispielen nicht in parenthetischem Gebrauch, sondern satzintegriert auf. Auch in diesem Fall scheint die Verwendung textsortenabhängig zu sein.

(iii) In der Funktion „Fokusdomäne“ scheint auch in der mündlichen Interaktion zahlenmäßig stark vertreten zu sein (32/110). In dieser Verwendung wird die Grundbedeutung von auch, nämlich die Operation des Addierens selbst, hervorgehoben. Dies ist im Italienischen mit Bezug auf anche kaum vertreten.

(iv) Auch und anche in der Funktion „Modalität” treten in unserem Korpus nur in Deklarativsätzen auf. Was das Deutsche betrifft, liegt dies daran, dass die anderen Satzmodi, in denen auch in dieser Funktion auftreten kann, stark markierte Sprechhandlungen (Aufforderungen und Vorwürfe) realisieren, die dementsprechend in informellen Unterhaltungsgesprächen seltener vollzogen werden. Was anche angeht, ist sein modales Verwendungsspektrum von vorneherein eingeschränkter. Unsere Daten bestätigen, dass modales anche nur in Deklarativsätzen und in der Regel in Kombination mit einem Modalverb möglich ist.

Zusammenfassend lässt sich anhand der Punkte (i) bis (iv) noch einmal bestätigen, dass - obwohl alle vier Funktionen für beide Partikeln vertreten sind - bei anche die additive Funktion eindeutig vorherrschend ist. Dies hängt damit zusammen, dass anche syntaktisch an seinen Bezugsbereich linksadjazent sein und nicht in einer bestimmten Position im Satz realisiert werden muss. Dagegen zeigen unsere Daten, dass auch präferiert im Mittelfeld auftritt, was alle Interpretationen zulässt. Von dieser Position aus kann die Partikel auch nicht nur auf syntagmatischer, sondern auch auf textueller (Satzverknüpfung), pragmatischer (Fokusdomäne) und modaler Ebene operieren. 


\section{Literaturverzeichnis}

Abraham, Werner (2020): Modality in Syntax, Semantics, and Pragmatics. Oxford: Oxford University Press.

Andorno, Cecilia (1999): „Avverbi focalizzanti in italiano. Parametri per un'analisi”. Studi italiani di linguistica teorica e applicata 28: 43-83.

Andorno, Cecilia (2000): Focalizzatori fra connessione e messa a fuoco. Il punto di vista delle varietà di apprendimento. Milano: Franco Angeli.

Andorno, Cecilia (2008): „Ancora su anche, anche su ancora. Per uno studio comparativo dell'apprendimento e della gestione di strategie coesive in L2“. In: Romano Lazzeroni et al. (eds.): Diachronica et Synchronica. Studi in onore di Anna Giacalone Ramat. Pisa, ETS Edizioni: 29-52.

Benazzo, Sandra/Dimroth, Christine (2015): „Additive Particles in Romance and Germanic Languages: Are They Really Similar?" Linguistik online 71: 9-30.

Benazzo, Sandra/Paykin, Katia (2017): „Additive relations in L2 French: Contrasting acquisitional trends of Italian and Russian learners". In: De Cesare, Anna-Maria/Andorno, Cecilia (eds.): Focus on Additivity. Adverbial modifiers in Romance, Germanic and Slavic languages. Amsterdam/Philadelphia, Benjamins: 265-309.

Bidese, Ermenegildo et al. (2019): „The expression of additivity in the Alps: German auch, Italian anche and their conuterparts in Gardenese Ladin". In: Bidese, Ermenegildo/Casalicchio, Jan/Moroni, Manuela Caterina (eds.): La linguistica vista dalle Alpi - teoria, lessicografia e multilinguismo. Studi in onore di Patrizia Cordin/Linguistic views from the Alpslanguage theory, lexicography and multilingualism. Studies in Honor of Patrizia Cordin. Berlin etc., Lang: 339-370.

Borreguero Zoulaga, Margarita (2012): „Focalizzatori nelle varietà di apprendimento: il caso di 'anche"'. In: Bianchi, Patricia et al. (eds.): La variazione nell'italiano e nella sua storia. Varietà e varianti linguistiche e testuali. Atti dell'XI Convegno della Società Internazionale di Linguistica e Filologia Italiana. (Napoli, 7-10 ottobre 2010). Firenze, Franco Cesati: 617-628.

Burkhardt, Armin (1985): „Der Gebrauch der Partikeln im gesprochenen Deutsch und im gesprochenen Italienisch". In: Holtus, Günter/Radtke, Edgar (eds.): Gesprochenes Italienisch in Geschichte und Gegenwart. Tübingen, Narr: 236-275.

Bußmann, Hadumod (2002): Lexikon der Sprachwissenschaft. Stuttgart: Kröner.

Coniglio, Marco (2008): „Modal particles in Italian”. University of Venice working papers in linguistics 18: 91-129.

Coniglio, Marco (2011): Die Syntax der deutschen Modalpartikeln: ihre Distribution und Lizenzierung in Haupt- und Nebensätzen. Berlin: Akademie Verlag.

De Cesare, Anna-Maria (2004): „L'avverbio anche e il rilievo informativo del testo”. In: Ferrari, Angela (ed.): La lingua nel testo, il testo nella lingua. Torino, Istituto dell'Atlante Linguistico Italiano: 191-218.

De Cesare, Anna-Maria (2015): ,Additive focus adverbs in canonical word orders. A corpusbased study of It. anche, Fr. aussi and E. also in written news". Linguistik online 71: 3156.

Diewald, Gabriele (2011): „Pragmaticalization (defined) as grammaticalization of discourse functions". Linguistics 49: 365-390. 
Dimroth, Christine (2004): Fokuspartikeln und Informationsgliederung im Deutschen. Tübingen: Stauffenburg.

Duden (2016): Duden - Die Grammatik: unentbehrlich für richtiges Deutsch. Herausgegeben von Angelika Wöllstein und der Dudenredaktion. 9. Aufl. Berlin: Dudenverlag.

Frascarelli, Mara/Hinterhölzl, Roland (2007): „Types of Topics in German and Italian”. In: Schwabe, Kerstin/Winkler, Susanne (eds.): On Information Structure, Meaning and Form. Amsterdam/Philadelphia, Benjamins: 87-116.

Held, Gudrun (1985): „Ma, dico, sei proprio dura, eh! - Zu Formen und Funktionen einiger lexikalischer Verstärkungsmittel in Dialogreaktionen”. In: Holtus, Günter/Radtke, Edgar (eds.): Gesprochenes Italienisch in Geschichte und Gegenwart. Tübingen, Narr: 300-328.

Kempowsky, Walter (1982): Tadellöser \& Wolff. Ein bürgerlicher Roman. München: Carl Hansa Verlag.

Kleemann, Anja (2007): „The Syntax of Focus Particles in German Event vs. Result Nominals“. In: Chang, Charles et al. (eds.): CamLing 2006: Proceedings of the 4th University of Cambridge Postgraduate Conference in Language Research. Cambridge, UK, Cambridge Institute of Language Research: 100-107.

Pasch, Renate et al. (2003): Handbuch der deutschen Konnektoren. Bd. 1. Berlin/New York: de Gruyter.

Radtke, Edgar (1985): „Abtönungsverfahren im gesprochenen Italienisch. Zu magari, figurati und Rekurrenzstrategien". In: Holtus, Günter/Radtke, Edgar (eds.): Gesprochenes Italienisch in Geschichte und Gegenwart. Tübingen, Narr: 280-299.

Reis, Marga/Rosengren, Inger (1997): A Modular Approach to the Grammar of Additive Particles: the Case of German auch. Journal of Semantics 14: 237-309.

Ricca, Davide (1999): „Osservazioni preliminari sui focalizzatori in italiano”. In: Dittmar, Norbert/Giacalone Ramat, Anna (eds.): Grammatica e discorso. Studi sull'acquisizione dell'italiano e del tedesco. Tübingen, Stauffenburg: 146-164.

Sabatini, Francesco/Coletti, Vittorio (1997): DISC: Dizionario Italiano Sabatini Coletti. Firenze: Giunti.

Selting, Margret et al. (2009): Gesprächsanalytisches Transkriptionssystem 2 (GAT2). Gesprächsforschung - Online Zeitschrift zur verbalen Interaktion 10: 353-402.

Siegl, Elfie (1997): Russischer Bilderbogen. Reportagen aus einem unbegreiflichen Land. Hamburg: Rotbuch Verlag.

Thurmair, Maria (1989): Modalpartikeln und ihre Kombinationen. Tübingen: Niemeyer.

\section{Korpora}

DeReKo: Deutsches Referenzkorpus Institut für Deutsche Sprache Mannheim. ids-mannheim.de/kl/projekte/korpora/ [02.11.2021].

FOLK: Forschungs- und Lehrkorpus gesprochenes Deutsch Datenbank für Gesprochenes Deutsch (DGD) Institut für Deutsche Sprache Mannheim. dgd.ids-mannheim.de [02.11.2021].

LIP: Lessico di frequenza dell'italiano parlato. badip.uni-graz.at/it/corpus-lip/descrizione [02.11.2021]. 


\section{Online Wörterbücher}

Corriere della sera dizionario online: Corriere della Sera, Milano (2021). dizionari.corriere.it/dizionario_tedesco/index.shtml [01.06.2021]

Dizionario Hoepli Tedesco-Italiano: HOEPLI S.p.A., Milano. grandidizionari.it/Dizionario_Italiano-Tedesco.aspx?idD $=6$ [01.06.2021]

Langenscheidt Dizionari online: PONS GmbH, Stuttgart. it.langenscheidt.com/tedescoitaliano/ [01.06.2021].

Pons Dizionario Online: PONS GmbH, Stuttgart. it.pons.com/traduzione [01.06.2021].

\section{Weitere Internetquellen}

Forumeintrag: Ben: „Re: nuovo arrivo (sia ringraziato che ci sono arrivato!! An“, 16.9.2010: 11:09 am. Promiseland.it. VEGANOK SRL SOCIETA' BENEFIT. https:/forum.promiseland.it/viewtopic.php? $\mathrm{f}=28 \& \mathrm{t}=32468 \&$ start $=585 \&$ view $=$ print $[01.06 .2021]$. 